\title{
Trade-off between mate choice speed and decision accuracy under mating competition in female sand gobies
}

\author{
Beatriz Diaz Pauli ${ }^{1}$ (D) Kai Lindström ${ }^{2}$
}

Received: 18 December 2019 / Accepted: 20 August 2020 / Published online: 2 September 2020

(c) The Author(s) 2020

\begin{abstract}
Variation in female mating performance can affect the direction and rate of evolution through sexual selection. The social environment determines the availability of mates and the competitive situation, and hence can influence mating strategies. However, these effects have to be considered within time constraints. Here we show that female sand gobies exposed to samesex competitors (female-biased adult sex ratio, ASR) for a week before having physical access to males (i.e., a delayed male access; referred as prior exposure treatment) were more actively associated with the preferred male and took faster spawning decisions. However, these females mated more frequently with males with traits that did not ensure high egg survival. On the other extreme, females exposed to low same sex competition (male-biased ASR) simultaneously to the mate choice (i.e., an immediate access to males; referred as simultaneous exposure treatment) took more time to make their spawning decision. They also associated and spawned more often with males with good parental skills (i.e., males exhibiting more egg fanning behaviour). These male traits are associated with higher survival and better development of eggs. Our results provide experimental support for a trade-off between mate choice accuracy and speed, which depended on mating competition.
\end{abstract}

Keywords Mate choice $\cdot$ Adult sex ratio $\cdot$ Parental care $\cdot$ Mating decision $\cdot$ Mate sampling $\cdot$ Pomatoschistus minutus

\section{Introduction}

Variation in mating behaviour can affect evolution through sexual selection (Jennions and Petrie 1997), which depends on intersexual choice and intrasexual competition (Darwin 1871; Andersson 1994). In many species, changes in the social environment, i.e., availability of mates and competitors for those mates, can occur quickly in space and time (Forsgren et al. 2004; Ogburn et al. 2014), and lead to different behavioural responses (Kokko and Rankin 2006; AhKing and Gowaty 2016). Studies on the context-dependency of female mate choice have focused on either preference for male traits or the time constraints for female mate sampling, but the combination of both are seldom considered

Beatriz Diaz Pauli

Beatriz.Diaz-Pauli@uib.no

Kai Lindström

Kai.Lindstrom@abo.fi

1 Department of Biological Sciences, University of Bergen, 5020 Bergen, Norway

2 Environmental and Marine Biology, Åbo Akademi University, 20500 Turku, Finland
(Lindström and Lehtonen 2013; Breedveld and Fitze 2015; Henshaw 2018), despite the acknowledged importance of time responses in mate choice (Jennions and Petrie 1997; Castellano et al. 2012; Edward 2015).

Our still limited knowledge on how females and males make their mating decisions, would be enhanced by incorporating time constraints into the widely studied contextdependency of female mating decisions (Castellano et al. 2012). Females obtain clear benefits from mating with high quality males that offer higher fitness to the female and/ or the offspring (Reynolds and Gross 1990). Search cost in females include increased energy and time expenditure, increase predation risk and loss of mating opportunities while mate sampling (Forsgren 1997a). Time is an important component in mate choice, because a female can spend longer time assessing mate quality, but this gathering of information comes at the cost of reduced lifetime reproductive success (i.e., missing mating opportunities; Sullivan 1994; Henshaw 2018). Thus, time constraints in mate choice, as in many animal decision making situations, can result in a speed-accuracy trade-off (Wickelgren 1977; Chittka et al. 2009). According to such a trade-off, accuracy could be gained by investing longer time in taking the 
decision and faster decisions would imply more errors or lower value rewards (Pirrone et al. 2014). Speed-accuracy trade-offs could also play a role in the context of mate choice and possibly result in a weakened relationship between mate choice traits and fitness.

Here we studied how the interplay between competition for mates and delayed access to mates (i.e., the duration of time before a female was allowed physical access to males) affected female choice in the sand goby, Pomatoschistus minutus, a small sexually dimorphic fish with parental care. Sand goby females prefer males with good parental skills (Forsgren 1997a; Lindström et al. 2006). An earlier study showed female sand gobies made faster mating decisions when the access to mates was delayed, i.e., the cost of mate sampling was increased (Lindström and Lehtonen, 2013). However, the accuracy of the mating decision was not assessed.

In the current study, we manipulated the adult sex ratio (ASR) and the timing of the access to mates to evaluate their effect on female mate choice performance. ASR refers here to the ratio of adult males to females, which drives variation in the ratio of sexually active males to receptive females (Emlen and Oring 1977). We exposed females to either a male or female biased ASR. The period of exposure to the ASR was either simultaneous to the mate access or for one week prior to the access to mates. Therefore, the timing of the access to mates was either immediate or delayed relative to the first mate encounter. We categorised males according to their size and success in male-male competition for nests before the mate choice test, and mating success and parental care after the mate choice test. We evaluated female choice towards these male attractive traits in the different scenarios. We expected females to take faster decisions when the cost of mating, in terms of reduced future chances of reproductive success was high. This would occur at high female competition (female-biased ASR) and/or when the access to mates was delayed by exposing females to the ASR 1 week prior the mating opportunity. Faster mating decision could lead to a speed-accuracy trade-off, a loss of preference or a shift in male traits preferred.

\section{Materials and methods}

\section{Study area}

This study was carried out at Tvärminne Zoological Station, Southern Finland during the sand goby breeding season (May-July) in 2007. Fish were collected from a natural breeding area by hand trawl and by hand netting individuals from previously provided artificial nests. Fish were housed in separate-sex holding tanks prior to the experiment. Fish were fed ad libitum with live Mysis and frozen chironomid larvae. At the end of the experiment all fish were released back to where they were caught.

\section{Adult sex ratio and timing of mate access}

The social context experienced by the focal females was modified by two factors: the adult sex ratio (ASR, femalebiased vs. male-biased) and the timing of exposure to the ASR (prior vs. simultaneous exposure periods) relative to the physical access to the mate resulting in a $2 \times 2$ factorial design (Fig. 1). Total sample size was $N=78$ focal females, but it was not perfectly balanced among treatments (femalebiased prior encounter: $N=19$, female-biased simultaneous encounter: $N=20$, male-biased prior encounter: $N=19$, and male-biased simultaneous encounter: $N=20$ ). The total number of focal males was 156. The female-biased ASR consisted of two males and four females (the focal and three stimulus females), while the male-biased ASR treatment consisted of four males and two females (the focal and one stimulus female; Fig. 1b). The female-biased ASR thus represented higher same-sex competition for the focal female and a cost in terms of limited mating opportunities. Stimulus fish were additional fish needed for the different ASR (males and females) and were used only once before being released back to sea. Thus, stimulus fish were not used as focal or again as stimulus fish in later replicates. Focal females were chosen randomly from all the females available during the prior exposure period.

In the prior exposure period, the focal female was exposed to the ASR treatment during 1 week before the mate choice test and actual physical access to mates, but the ASR treatment was removed during the mate choice test. Thus, the female-biased (M:F=2:4) and male-biased ASR (M:F=4:2) during the 1-week exposure period became a male-biased ASR (M:F=2:1) during the mate choice test. In the simultaneous exposure period, the focal female experienced the ASR treatment simultaneously with the mate choice test. In the prior exposure period, the males and focal and stimulus females had only visual contact to avoid spawning. Thus, in the prior exposure period, focal females encountered potential mates 1 week before they had direct access to mates. In the simultaneous exposure treatment, the focal females came directly from a female-only stock tank. Therefore, they encountered and had access to the males simultaneously and immediately when the experiment started. Each of the stimulus males was given a halved clay flowerpot (diameter $4 \mathrm{~cm}$ ) as nest site material and that could house the egg clutch of one female, maybe two. All females used were ready to spawn as determined by their belly roundness (García-Berro et al. 2019). All males were also ready to mate, as they all owned, built and defended a nest (see e.g., Forsgren 1999; Malavasi et al. 2001). 


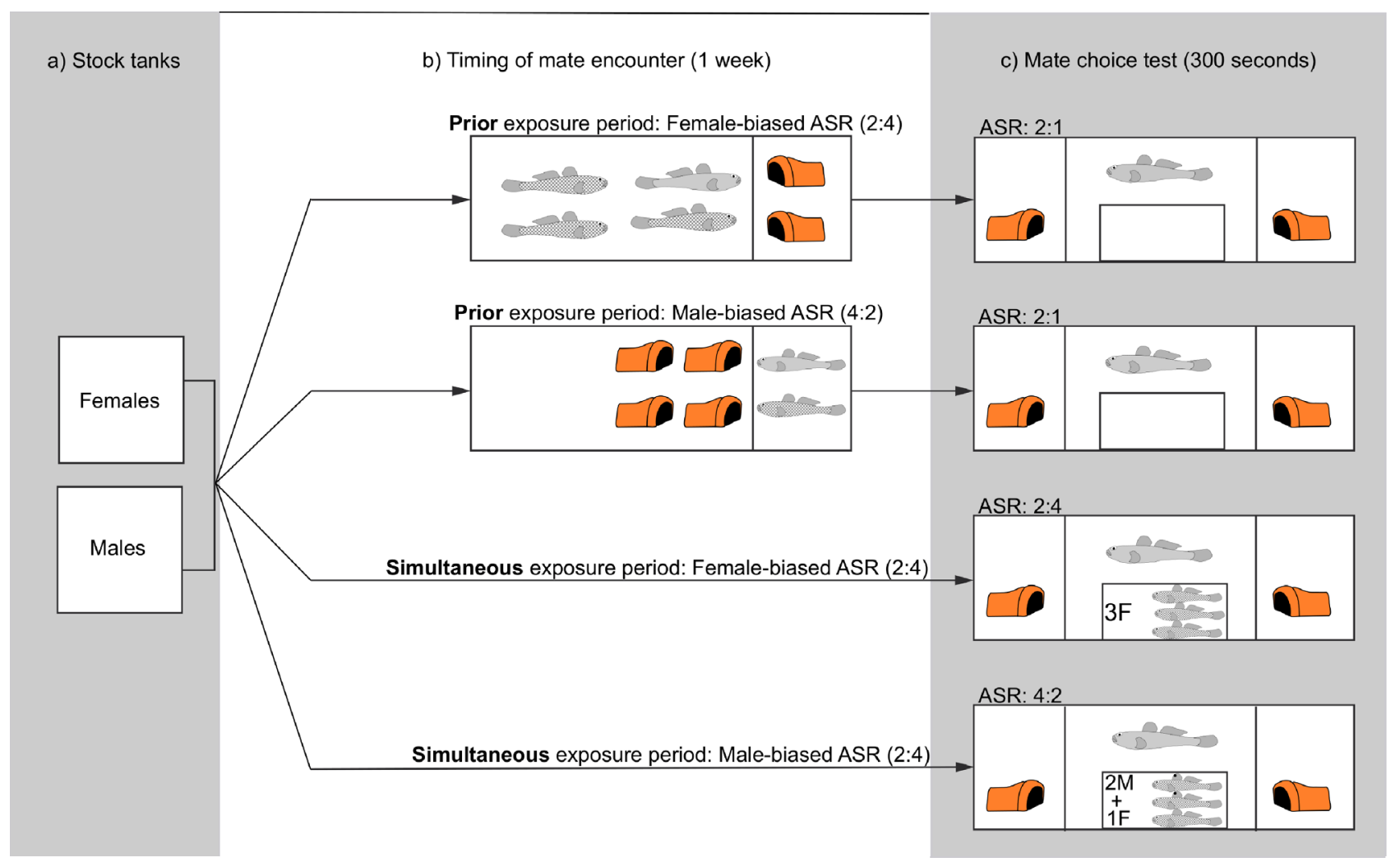

Fig. 1 Experimental set-up showing the $2 \times 2$ factorial design and the different phases in the experiment from a) stocking the fish, b) timing of mate encounter and c) mate-choice test. The prior exposure treatment shows that the focal female (grey fish) first encountered potential mates for one week prior to the mate-choice test and spawning opportunity (i.e., delayed access to mates), while in the simultaneous exposure treatment the first mate encounter occurs simultaneously to the mate-choice test and spawning opportunity (i.e., immediate

\section{Mate choice tests}

Experimental aquaria $(70 \times 40 \times 25 \mathrm{~cm}$ length $\mathrm{x}$ height $\mathrm{x}$ depth) were exposed to daylight and had a continuous flowthrough of seawater. Hence light, temperature and salinity followed natural conditions. The tanks were divided into three compartments (Fig. 1). The two smaller side compartments housed a male on each side and their nests (halved clay flowerpots, $4 \mathrm{~cm}$ diameter). These males were considered focal males and were never used as stimulus males earlier or later in the experiment. The two males were the dominant and the subordinate male from a nest competition challenge (male status: dominant vs. subordinate). The dominant male was the winner out of three randomly selected males competing for a single nest site. The subordinate male was the male left with no nest after the dominant had been removed and a second nest was provided to the two remaining males.

The two focal males in the mate choice test were separated by opaque dividers and were given $24 \mathrm{~h}$ for nest access to mates). Female-biased ASR consists of four females (focal female represented in grey and three stimulus females in white) and two males (represented by their nests), while male-biased ASR is two females (focal female in grey and stimulus in white) and four males (represented by two nests and two stimulus males in white). $3 \mathrm{~F}$ clarifies that there are three stimulus females in box and $2 \mathrm{M}+1 \mathrm{~F}$ clarifies that the stimulus fish in box are two males and one female

building. A focal female, from either the prior or simultaneous ASR exposure period (i.e., exposed to ASR for a week or coming directly from female-only tank), was then introduced into the central section of the tank $(30 \times 25 \mathrm{~cm})$ in the morning (9:00 $\mathrm{AM} \pm 30 \mathrm{~min}$ ). In addition, a transparent box containing the stimulus fish (simultaneous exposure period) or empty (prior exposure period) was introduced into the central compartment to create the appropriate ASR. The male-biased ASR consisted of four males (the two focal males and two stimulus males) and two females (the focal and one stimulus female), while the female-biased ASR consisted of the two focal males and four females (the focal and three stimulus females; Fig. 1c.).

After $10 \mathrm{~min}$, the opaque dividers were removed and the female could see, but not physically interact with the two males. Female behaviour was observed for $15 \mathrm{~s}$, every $5 \mathrm{~min}$, 20 times, until a total observation time of $300 \mathrm{~s}$ was reached. The observations were made live and the observer was blind to the ASR treatment, as number of males and females could not be disentangled. However, at times it was possible to tell 
whether the stimulus box was empty or not, but not always as sand gobies occasionally bury themselves in the sand. We recorded the time the focal female spent within a fish length's distance from and oriented towards each male, i.e., time associated with a male. In addition, we recorded the time the female showed no preference for either male, as the time she spent in the middle of the tank. After recording behaviour, the transparent dividers were removed allowing the female access to the males (at 11:00 $\mathrm{AM} \pm 30 \mathrm{~min}$ ). The tanks were checked every hour for spawning until the next day at 16:00 PM (29 h). Once spawning had started, the focal female was enclosed together with the male she started spawning with in the male's compartment using an opaque divider to avoid being disturbed by the rejected male. When the female had finished spawning and left the nest, she was immediately removed from the tank. The chosen male was designated the 'preferred' male type, while the non-chosen male was designated male type 'rejected'. A female from the female holding tank was then added to the rejected male and given $24 \mathrm{~h}$ for spawning. This was done to induce parental care behaviour of the rejected male. Each nest was lined with a transparent film onto which eggs were spawned and which allowed photographing and counting the eggs.

The following day, paternal care was recorded (live and blind to the treatments from 9:00 to 12:00) as the time spent fanning the eggs during a 10-min period following earlier protocols (Lissåker and Kvarnemo 2006; Järvi-Laturi et al. 2008). Not all males received eggs or eggs were lost due to cannibalism. These males without eggs did not perform parental care and hence they were removed from the parental care analysis. Total sample size of parental care analyses was $N=52$ (female-biased prior encounter: $N=13$, femalebiased simultaneous encounter: $N=15$, male-biased prior encounter: $N=9$, and male-biased simultaneous encounter: $N=15$ ). Males were characterised by three independent male traits: 1) male body size (standard length), 2) male status, i.e., dominance vs. subordinance in the nest competition test, and 3) male type, i.e., preferred vs. rejected by the focal female shown by her mating decision.

Female condition (Fulton's condition index, fresh weightto-length $^{3}$ ratio) did not differ between ASR treatments (LMM: $F=1.92, P=0.39, N=100$ (52 focal females +48 females of rejected male), the time of ASR exposure period (LMM: $F=0.01, P=0.98, N=100$ ) and whether they were focal females or extra females added to the males rejected by the focal female (LMM: $F=0.002, P=0.97, N=100$ ). Male body size did not differ between ASR treatment (LMM: $F=0.07 P=0.79, N=156$ ), between time of exposure treatment (LMM: $F=0.23, P=0.63, N=156$ ), and between preferred and rejected males (LMM: $F=2.22, P=0.13$, $N=104)$. However, dominant males were on average $2.4 \mathrm{~mm}$ larger than subordinate males (LMM: $F=7.13, P=0.009$, $N=156)$.

\section{Statistical analyses}

Statistical analyses were performed in R statistical software (version 3.2.2; R Core Team, 2018). Female time associated with each male (continuous square-root-transformed data, seconds), female time showing no preference (continuous data, seconds), and male fanning time (continuous squareroot-transformed data, seconds) were analysed with linear mixed models (LMM). All models included female identity nested within tank identity as a random factor, except female time showing no preference, which only had tank identity as a random effect. Fixed factors were time of ASR encounter (prior vs. simultaneous), ASR (female- vs. male-biased), and male type (preferred by female vs. rejected), male status (dominant vs. subordinate), or body size. LMMs were performed with R package"lme4" (version 1.1-11; Bates et al. 2015). Latency to spawn was tested using survival analysis (cox-proportional hazards model; SA) with tank identity as random factor, and ASR, time of ASR encounter and time associated with males as fixed effects ("coxme" package, version 2-2.5 Therneau, 2018;). The 52 focal females were included in the latency to spawn analysis. Females who spawned were described by a censoring score of 1 and the time it took them to spawn in hours. Fixed-effects structure was simplified with the aid of Akaike's information criterion. The model that yielded the lowest AIC was considered the model that best described the data (Burnham and Anderson 2002). $P$ values were calculated from $F$ statistics for LMM (lmerTest package 2.0-29 Kuznetsova et al. 2017;). Pairwise comparisons were carried out with emmeans package (version 1.2.4), which compares estimated marginal means (EMMs) from the models (Lenth 2018).

\section{Results}

\section{Female preference}

Females spent more time associated with the male they eventually mated with (LMM: $F=13.06, P=0.0005, N=52$ ), but this was affected by the 3 -way interaction of ASR, time of ASR exposure, and male type (LMM: $F=10.37, P=0.002$, $N=52$ ). Only females exposed to the female-biased ASR in the prior exposure treatment and those exposed to the malebiased ASR in the simultaneous exposure treatment showed a clear association with the male they later spawned with (i.e., preferred male; Fig. 2). Females exposed to the femalebiased ASR and prior exposure treatment spent $58 \pm 2.9$ $(\mathrm{X} \pm \mathrm{SE})$ times longer with their preferred male relative to the rejected male (EMM: $t$-ratio $=4.45, P<0.001$; Fig. 2). Females exposed to the male-biased ASR and simultaneous exposure treatment spent $19 \pm 2.5(\mathrm{X} \pm \mathrm{SE})$ times longer with the preferred male (EMM: $t$-ratio $=2.72, P=0.009$ ). 


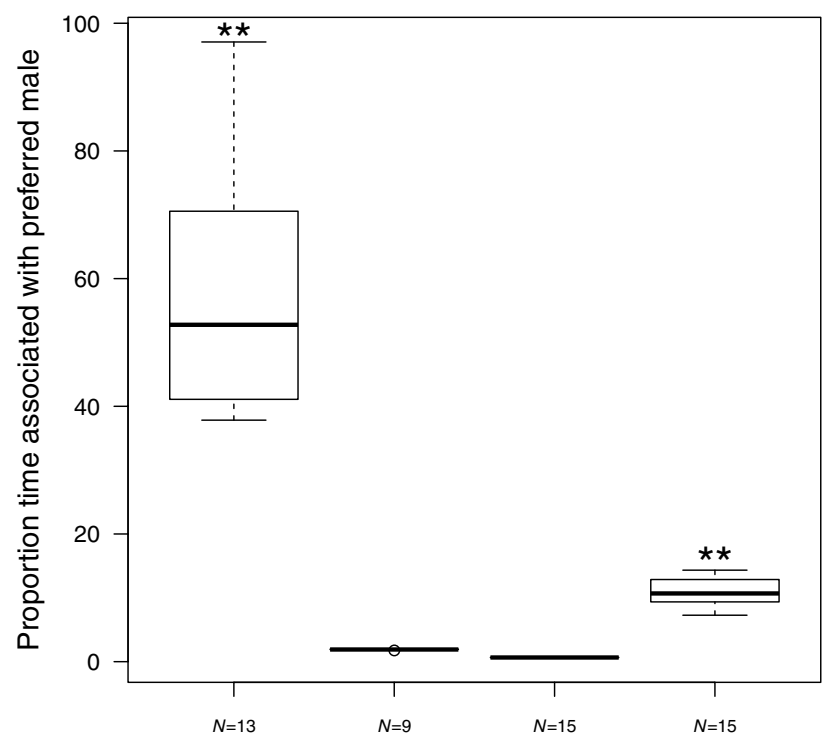

Female-biased Male-biased Female-biased Male-biased

Prior

Simultaneous

Fig. 2 Proportion of time the focal female is associated with the preferred compared to the rejected male. The different boxes represent ASR treatment (female- and male-biased) and the timing when the females were exposed to the ASR treatment (prior and simultaneous). Thick bars represent medians and the lower and upper hinges of the box represent first and third quartile, while whiskers show 1.5 time the interquartile range. $* *$ mark the groups where there was as significant difference $(P<0.01)$ between the preferred and rejected males

(a)

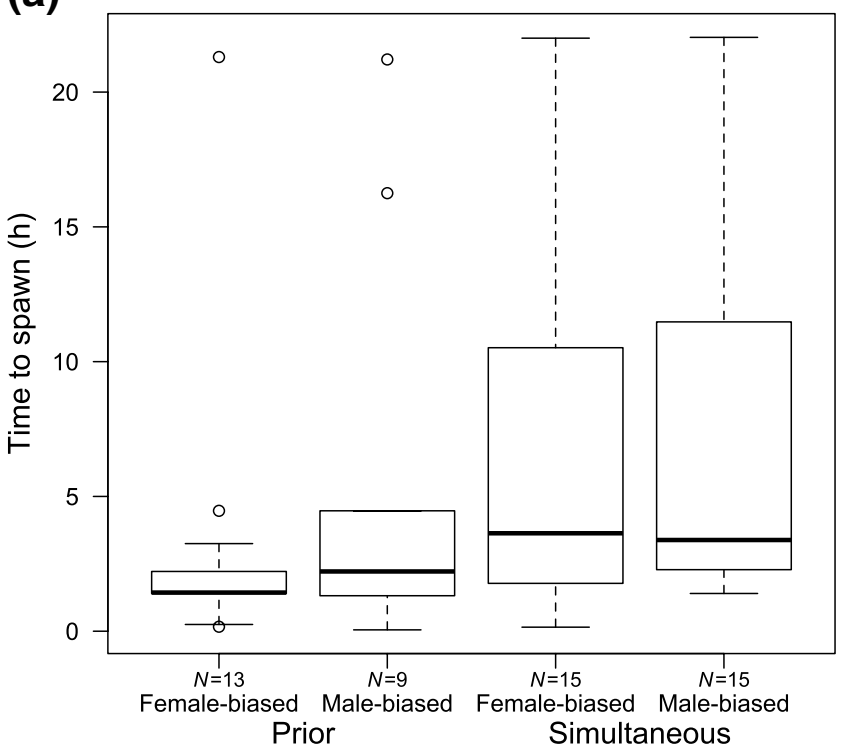

Fig. 3 a) The time it took the focal female to spawn with her preferred male in each treatment group (raw data). The different boxes represent ASR treatment (female- and male-biased) and the timing when the females encountered the ASR (prior and simultaneous). Thick bars represent medians, lower and upper hinges of the box represent first and third quartile, while whiskers show 1.5 time the interquartile range, and open circles represent observations outside the 1.5
Females exposed to the remaining two treatments showed no interest for either male (nor the stimulus box), as they spent most of their time in the middle of the tank as shown by the interaction between ASR and the time of ASR encounter (LMM: $F=4.33, P=0.041, N=78$ ). For instance, females that showed the least interest for males (i.e., those exposed to female-biased ASR and simultaneous exposure treatment) spent $55 \pm 23.7(\mathrm{X} \pm \mathrm{SE})$ times longer in the central compartment relative to females that showed the highest preference (i.e., female-biased and prior exposure treatment, EMM: $t$-ratio $=2.31, P=0.024)$. In contrast, females did not spend more time associated with either dominant or subordinate males (LMM: $F=0.40, P=0.530, N=78$ ). Nor this was affected by ASR treatment (LMM: $F=0.53, P=0.466$, $N=78$ ) or ASR exposure period (LMM: $F=3.02, P=0.084$, $N=78$ ). Male body size also did not affect the time females spent associated with the males (LMM: $F=0.22, P=0.639$, $N=78$ ).

The latency to spawn (spawning time) was affected by the treatments. These differences are described by the differences in spawning rate, i.e., number of spawning events per time observed expected in each treatment group. Females exposed to the prior exposure treatment had a four times higher spawning rate relative to females exposed to the simultaneous exposure treatment (Fig. 3a; SA: $z=-2.40$, $P=0.016, N=52$ ). This is equivalent to an average spawning

(b)

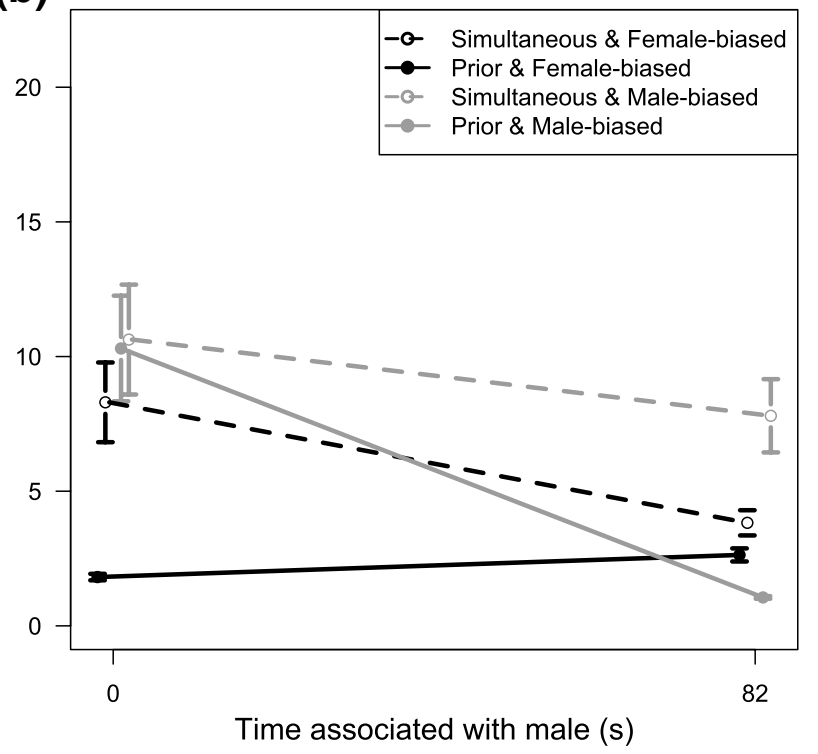

time interquartile range. b) Model estimated female time to spawn $(\mathrm{X} \pm \mathrm{SE}$ represented by dots and whiskers, respectively) linked to short $(0 \mathrm{~s})$ and long $(82 \mathrm{~s})$ female association time with her preferred males. The 0 and $82 \mathrm{~s}$ represent the $30^{\text {th }}$ and $70^{\text {th }}$ percentiles of time associated to preferred male, respectively $(X \pm S E=63.5 \pm 9.2 \mathrm{~s})$. Note dots are jittered to avoid overplotting 
time of $3 \mathrm{~h}$ earlier. Females exposed to the female-biased ASR had a 5.5 times higher spawning rate-i.e., they spawned on average $5 \mathrm{~h}$ earlier (Fig. 3a; SA: $z=-2.39$, $P=0.017, N=52)$. However, these effects depended on the time the female spent associated with the preferred male (Association time*ASR *Exposure period, SA: $z=-3.17$, $P=0.015, N=52$; Table 1; Fig. 3b). When we compared females that spent short times associated with the males they mated with, we found that females exposed to the femalebiased ASR and prior exposure treatment spawned on average five times faster than all the other groups (i.e., $7 \mathrm{~h}$ earlier), which did not differ from each other (Fig. 3b; Table 1). On the other hand, when the duration of the association time with the preferred male increased, the rate of spawning decreased in the female-biased and prior exposure group (Fig. 3b; Table 1). Moreover, with longer association times, females belonging to the male-biased simultaneous exposure group had the slowest spawning rate (Fig. 3b; Table 1).

\section{Difference between preferred and rejected males}

Males that successfully mated with the focal females (i.e., the preferred males) were those that spent on average $25 \mathrm{~s}$ more fanning their eggs than rejected males (LMM: $F=6.92$, $P=0.011, N=52$; Fig. 4 a). The latency to spawn was also associated with fanning time, but only in the female-biased ASR (Fig. 4b). Females exposed to the female-biased ASR and having longer spawning latencies mated with males that fanned 24.9 times more, relative to females making fast spawning decision (EMM: $t$-ratio $=-2.15, P=0.037$ ). In the male-biased ASR, females with long and short spawning latencies mated with males fanning equally much (EMM: $t$-ratio $=-0.89, P=0.375$ ). This suggests that, even though females always preferred males that performed more fanning behaviour, those females with faster spawning decisions (i.e., short latency to spawn) preferred and rejected good parents equally often and hence did not mate with good parents more frequently. While females with slow mating decisions (i.e., long latency to spawn) did end up mating with males of good parental quality. The exposure period (prior vs. simultaneous) was not associated with male fanning time when latency to spawn was included in the model. Finally, it should be noted that male status did not affect fanning behaviour. Dominant and subordinate males did not differ in their parental care behaviour (LMM: $F=0.91, P=0.350$, $N=52$, Fig. 4 a). In addition, male body size did not affect male fanning (LMM: $F=0.34, P=0.562, N=52$ ).

\section{Discussion}

In the present study we show that the timing (prior or simultaneous) when a female is exposed to a particular sex ratio environment (ASR treatment) affects her association with the male, with whom she later spawns and the latency to spawning, which we interpret as representing the speed of her decision making, rather than a change of preference. Thus, our results highlight the importance of considering the interplay between the availability of potential mates and the timing of meeting those mates to better understand female mating decision. This interaction is commonly not considered (Castellano et al. 2012; Breedveld and Fitze 2015).

Females exposed to the female-biased ASR for a week prior to the mate choice test exhibited the shortest spawning latency. These females also had the longest time associated with their preferred males, and accordingly the shortest time spent in the central compartment of the experimental tank showing no preference for either male. At the other extreme, females experiencing the male-biased ASR and simultaneous exposure period also exhibited strong preferences for
Table 1 Statistical results from pairwise comparison of groups associated 0 and $82 \mathrm{~s}$ with the preferred male $\left(30^{\text {th }}\right.$ and $70^{\text {th }}$ percentiles, respectively)

\begin{tabular}{llllrl}
\hline & Contrasts & Ratio & SE & $z$-ratio & $P$ \\
\hline At 0 s associ- & Prior and Female-b./Simultaneous and Female-b & 4.16 & 2.47 & 2.40 & 0.016 \\
ated with & Prior and Female-b./Prior and Male-b & 5.52 & 3.95 & 2.39 & 0.017 \\
preferred & Prior and Female-b./Simultaneous and Male-b & 5.78 & 3.63 & 2.79 & 0.005 \\
male & Simultaneous and Female-b./Prior and Male-b & 1.33 & 0.77 & 0.49 & 0.625 \\
& Simultaneous and Female-b./Simultaneous and Male-b & 1.39 & 0.63 & 0.73 & 0.465 \\
& Prior and Male-b./Simultaneous and Male-b & 1.05 & 0.64 & 0.08 & 0.939 \\
At 82 s asso- & Prior and Female-b./Simultaneous and Female-b & 1.38 & 0.73 & 0.61 & 0.542 \\
ciated with & Prior and Female-b./Prior and Male-b & 0.37 & 0.20 & -1.80 & 0.071 \\
preferred & Prior and Female-b./Simultaneous and Male-b & 2.71 & 1.11 & 2.44 & 0.015 \\
male & Simultaneous and Female-b./Prior and Male-b & 0.27 & 0.17 & -2.05 & 0.040 \\
& Simultaneous and Female-b./Simultaneous and Male-b & 1.97 & 1.02 & 1.31 & 0.189 \\
& Prior and Male-b./Simultaneous and Male-b & 7.38 & 4.14 & 3.56 & 0.0004 \\
\hline
\end{tabular}

Ratio and SE refers to the ratio of estimated spawning rates (number of spawning events per observed time) and standard errors of each contrast, with their associated and $P$ value 
(a)

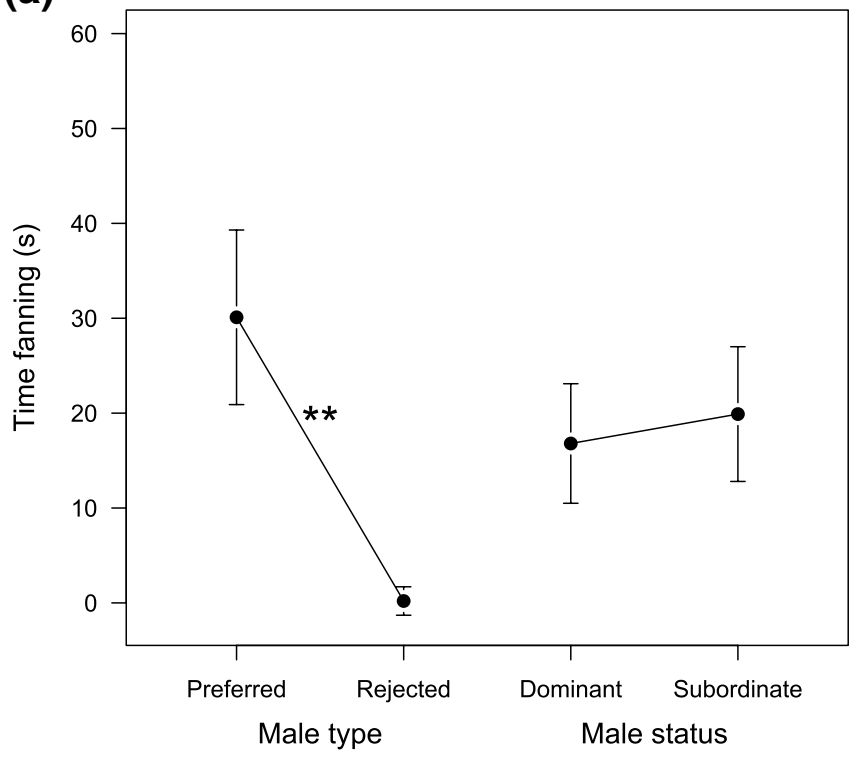

Fig. 4 a) Estimated time spent fanning the eggs by the preferred and the rejected males, and by dominant and subordinate males. Dots represent mean estimates while whiskers show standard errors. b) Estimated time spent fanning associated with short and long spawn-

one particular male by associating with that male longer than with the alternative male. However, in this case, the preference was not associated with faster spawning. Thus, it was the combination of a female-biased social environment (competition cost) and the delayed access to males (time cost) that resulted in faster spawning decisions (see also Lindström and Lehtonen 2013).

Interestingly, our results suggest a trade-off between the speed and accuracy of mate choice. Females with the highest mating costs (time and competition cost) took the fastest spawning decisions (i.e., female-biased ASR exposure prior to mate encounter), but did not always make an accurate choice, i.e., mated with the male with traits linked to better offspring survival. These females equally accepted and rejected males exhibiting good quality parental care. In sand gobies, females prefer males with good parental abilities (i.e., more egg fanning; Forsgren 1997b; Lindström et al. 2006), as these ensure better offspring survival and higher hatching success (Forsgren et al. 1996; Jones and Reynolds 1999; Klug et al. 2006; Karino and Arai 2006). Moreover, in the group exposed to a male-biased ASR simultaneous to mate encounter, females took longer to spawn and were most likely to mate with the male exhibiting most parental behaviour and hence made accurate decisions more frequently. It should be noted that we did not observe a shift in female preference towards other male traits. Females always preferred males with good parental skills across all the ASR conditions and ASR exposure periods. Our results showed that neither male size nor dominance in nest competition (b)

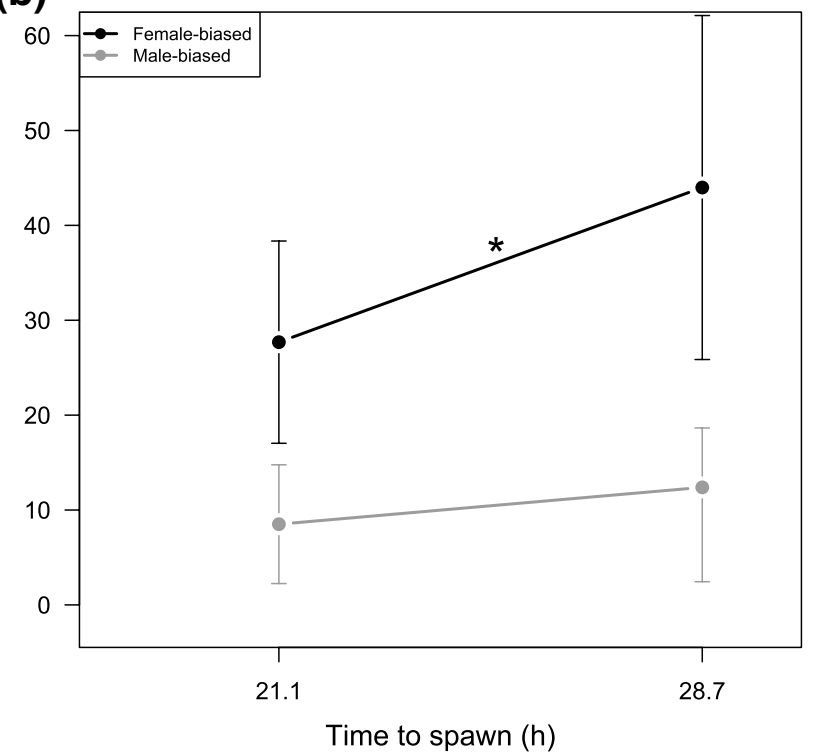

ing latencies for female- and male-biased ASR. The 21.1 and $28.7 \mathrm{~h}$ represent the $30^{\text {th }}$ and $70^{\text {th }}$ percentiles of latency to spawn, respectively $(\mathrm{X} \pm \mathrm{SE}=21.2 \pm 0.9 \mathrm{~h})$. $*$ and $* *$ mark significant differences of $P=0.04$ and $P=0.01$, respectively

became a preferred trait under any of the presented social environments. Dominant males were not better at parental care, confirming earlier results (Forsgren 1997b). Thus, the previous experience with female competitors and the time cost of delayed access to males led to a rushed mating decision that on average resulted in a worse choice of mate. Whether this was an erroneous choice or that females lowered their mate quality standards, as seen in other fish (Milinski and Bakker 1992; Heubel et al. 2008; Passos et al. 2014), cannot be determined by the present study. Either way, our results demonstrate a pronounced speed-value trade-off, which is equivalent to the previously suggested speed-accuracy trade-off (Chittka et al. 2009; Pirrone et al. 2014). It should be noted that the remaining two treatment groups lie within these two extremes. Females exposed to a male-biased ASR prior to mate choice, had the second shortest spawning latency and the difference in preferred and rejected males was the second smallest. Females exposed to a female-biased ASR simultaneously to mate choice had longer spawning latencies, but this did not result in relatively better mate choice decisions compared to females exposed to a male-biased ASR prior to mate choice. We believe that in this case, female-female competition led to a lowered mate selectivity to ensure any mating, as seen in other fish (Borg et al. 2006; Passos et al. 2014). This low selectivity was represented by a lack of a significant difference in association time between preferred and rejected males. In the rest of the groups, females exhibited a preference towards one of the males by associating with that male, and whether that 
preference resulted in mating with the high-quality male depended on spawning latency. Speed-accuracy trade-offs have been observed in decisions related to foraging, predator avoidance, in cognitive tasks in many animals (e.g., birds, bank voles and bees; Ducatez et al. 2015; Wang et al. 2018; Mazza et al. 2019) and even in unicellular mould (Dussutour et al. 2019). Similar trade-offs between time constraints and choice of the best mating partner are expected (Chittka et al. 2009), and have been found for example in the pied flycatcher, blue crabs, and house crickets (Alatalo et al. 1988; Backwell and Passmore 1996; Gray 1999). Our data suggest that this trade-off may be influenced by time constraints due to varying time of exposure to potential mates, rather than just time within a mating season, as the abovementioned studies have shown. In addition, we showed that the competitive setting, the previously experienced sex ratio, affected this trade-off.

All focal females in the present experiment experienced the same delay in their spawning opportunity with males, but females that experienced the prior exposure period could observe males with nests for a week before physical access and spawning opportunity. Females in the simultaneous ASR exposure period spent their time in all-female stock tanks and did not have any contact with males until the mate choice trials. Therefore, a possible interpretation of the difference between the two ASR exposure periods is that they represent different onsets of the females' mating window. Breedveld and Fitze (2015) define the start of the mating window with the first mate encounter. Thus, females in the prior exposure period could have initiated their mating window 1 week before while females in the simultaneous exposure period did not initiate their mating window until the mate choice test, when they encountered stimulus males for the first time (Breedveld and Fitze 2015; Henshaw 2018). Given this difference in the onset of the mating window, we believe that females in the prior exposure period experienced a time cost through missed mating opportunities, due to the delayed between first encounter and actual access to mates. These females hence experienced a higher time cost that affected their decisions than the females in the simultaneous exposure period (Breedveld and Fitze, 2015; Lindström and Lehtonen, 2013). The females maintained in the all-female stock tank (simultaneous exposure) may not have initiated the mating window until exposed to the mate choice test and did therefore not experience the cost of missed mating opportunities. The differences between females in the prior and the simultaneous exposures periods could be physiological. An earlier first mate encounter in the prior group may have stimulated earlier egg hydration (Tang et al. 2019) and thus resulted in over-mature eggs at the time of mate choice. Therefore, it may be this time constrain associated with deteriorating egg quality after ovulation and hydration that forces females to mate faster and with worse quality males (Goncalves et al. 2015). Differences between our ASR exposure period levels could also have been interpreted as differences in mate encounter rates by the females. The prior exposure treatment would represent a higher encounter rate relative to the simultaneous exposure. A higher mate encounter rate is expected to result in females being more choosy (Fawcett and Johnstone 2003), rather than less as observed here. However, the availability of males does not seem to affect spawning latency in sand gobies (Lindström and Lehtonen 2013). Therefore, we believe that differences in the onset of the mating window remains as a likely explanation for our results, although the existence of a mating window has not been investigated in sand gobies.

In conclusion, we showed that females with an extended experience of a female-biased ASR made faster spawning decisions. Under these conditions, female mating decisions were less accurate regarding male parental skills suggesting a trade-off between speed of mating decision and quality of mate choice.

Acknowledgements We thank Tvärminne zoological station for providing excellent research facilities and the entire sand goby team for discussions and help in the lab. Their suggestions are greatly appreciated. Funding was provided by the Research Council of Norway (to BDP, 275125), and the Academy of Finland (to KL, 211973).

Author contributions Both authors contributed to the study conception and design. Material preparation, data collection and analysis were performed by Beatriz Diaz Pauli. The first draft of the manuscript was written by Beatriz Diaz Pauli and both authors commented on previous versions of the manuscript. Both authors read and approved the final manuscript.

Funding Open Access funding provided by University of Bergen.

\section{Compliance with ethical standards}

Conflict of interest The authors declare that they have no conflicts of interest.

Ethical approval The study complies with all the relevant laws of Finland and was approved by Finnish authorities, permit no. HY 46-03 licensed by the University of Helsinki animal housing authorities. No harmful manipulations or invasive samples were performed in this study. Details on animal's origin, disposal, numbers and housing conditions are given in the methods section.

Data statement The data will be available on Mendeley data repository.

Open Access This article is licensed under a Creative Commons Attribution 4.0 International License, which permits use, sharing, adaptation, distribution and reproduction in any medium or format, as long as you give appropriate credit to the original author(s) and the source, provide a link to the Creative Commons licence, and indicate if changes were made. The images or other third party material in this article are included in the article's Creative Commons licence, unless indicated otherwise in a credit line to the material. If material is not included in the article's Creative Commons licence and your intended use is not 
permitted by statutory regulation or exceeds the permitted use, you will need to obtain permission directly from the copyright holder. To view a copy of this licence, visit http://creativecommons.org/licenses/by/4.0/.

\section{References}

Ah-King M, Gowaty PA (2016) A conceptual review of mate choice: stochastic demography, within-sex phenotypic plasticity, and individual flexibility. Ecology and Evolution 6:4607-4642

Alatalo RV, Carlson A, Lundberg A (1988) The search cost in mate choice of the pied flycatcher. Anim Behav 36:289-291. https:// doi.org/10.1016/S0003-3472(88)80272-0

Andersson M (1994) Sexual selection. Princeton University Press, Princeton, New Jersey

Backwell P, Passmore NI (1996) Time constraints and multiple choice criteria in the sampling behaviour and mate choice of the fiddler crab, Uca annulipes. Behav Ecol Sociobiol 38:407-416

Bates D, Maechler M, Bolker B, Walker S (2015) Fitting linear mixed-effects models using lme4. J Stat Softw 67:1-48

Borg ÅA, Forsgren E, Amundsen T (2006) Seasonal change in female choice for male size in the two-spotted goby. Anim Behav 72:763-771. https://doi.org/10.1016/j.anbehav.2005.11.025

Breedveld MC, Fitze PS (2015) A matter of time: delayed mate encounter postpones mating window initiation and reduces the strength of female choosiness. Behav Ecol Sociobiol 69:533-541

Burnham KP, Anderson DR (2002) Model selection and inference: A practical information-theoretical approach, 2nd edn. SpringerVerlag, New York

Castellano S, Cadeddu G, Cermelli P (2012) Computational mate choice: Theory and empirical evidence. Behav Processes 90:261-277

Chittka L, Skorupski P, Raine NE (2009) Speed-accuracy tradeoffs in animal decision making. Trends Ecol Evol 24:400-407

Darwin C (1871) The descent of man, and selection in relation to sex. Murray, London

Ducatez S, Audet JN, Lefebvre L (2015) Problem-solving and learning in Carib grackles: individuals show a consistent speed-accuracy trade-off. Anim Cogn 18:485-496. https://doi.org/10.1007/ s10071-014-0817-1

Dussutour A, Ma Q, Sumpter D (2019) Phenotypic variability predicts decision accuracy in unicellular organisms. Proc R Soc B 286:20182825. https://doi.org/10.1098/rspb.2018.2825

Edward DA (2015) The description of mate choice. Behav Ecol 26:301-310

Emlen ST, Oring LW (1977) Ecology, sexual selection, and evolution of mating syst. Science 197:215-223

Fawcett TW, Johnstone RA (2003) Mate choice in the face of costly competition. Behav Ecol 14:771-779. https://doi.org/10.1093/ beheco/arg075

Forsgren E (1997a) Mate sampling in a population of sand gobies. Anim Behav 53:267-276

Forsgren E (1997b) Female sand gobies prefer good fathers over dominant males. Proc R Soc B 264:1283-1286

Forsgren E (1999) Sexual selection and sex roles in the sand goby. In: Almada CV, Oliveira RF, Gonçalves EJ (eds) Behaviour and conservation of littoral fishes. Instituto Superior de Psicologia Aplicada, Lisbon, pp 249-274

Forsgren E, Amundsen T, Borg AA, Bjelvenmark J (2004) Unusually dynamic sex roles in a fish. Nature 429:551-554
Forsgren E, Karlsson A, Kvarnemo C (1996) Female sand gobies gain direct benefits by choosing males with eggs in their nests. Behav Ecol Sociobiol 39:91-96

García-Berro A, Yliportimo J, Lindström K, Kvarnemo C (2019) Understanding resource driven female-female competition: ovary and liver size in sand gobies. R Soc Open Sci 6:190886. https://doi.org/10.1098/rsos.190886

Goncalves IB, Mobley KB, Ahnesjö I et al (2015) Effects of mating order and male size on embryo survival in a pipefish: Maternal and Paternal Effects. Biol J Linn Soc Lond 114:639-645. https ://doi.org/10.1111/bij.12441

Gray DA (1999) Intrinsic factors affecting female choice in house crickets: Time cost, female age, nutritional condition, body size, and size-relative reproductive investment. J Insect Behav 12:691-700

Henshaw JM (2018) Finding the one: optimal choosiness under sequential mate choice. J Evol Biol 31:1193-1203. https://doi. org/10.1111/jeb.13296

Heubel KU, Lindström K, Kokko H (2008) Females increase current reproductive effort when future access to males is uncertain. Biol Lett 4:224-227

Järvi-Laturi M, Lehtonen TK, Pampoulie C, Lindström K (2008) Paternal care behaviour of sand gobies is determined by habitat related nest structure. Behaviour 145:39-50. https://doi. org/10.1163/156853908782687197

Jennions MD, Petrie M (1997) Variation in mate choice and mating preferences: a review of causes and consequences. Biol Rev 72:283-327

Jones JC, Reynolds JD (1999) Costs of egg ventilation for male common gobies breeding in conditions of low dissolved oxygen. Anim Behav 57:181-188. https://doi.org/10.1006/ anbe. 1998.0939

Karino K, Arai R (2006) Effect of clutch size on male egg-fanning behavior and hatching success in the goby, Eviota prasina (Klunzinger). J Exp Mar Biol and Ecol 334:43-50

Klug H, Lindström K, St Mary CM (2006) Parents benefit from eating offspring: density-dependent egg survivorship compensates for filial cannibalism. Evolution 60:2087-2095

Kokko H, Rankin DJ (2006) Lonely hearts or sex in the city? Density-dependent effects in mating systems. Philos T R Soc B 361:319-334

Kuznetsova A, Brockhoff PB, Christensen RHB (2017) lmerTest Package: Tests in Linear Mixed Effects Models. J Stat Softw 82:1-26. https://doi.org/10.18637/jss.v082.i13

Lenth R (2018) emmeans: Estimated Marginal Means, aka LeastSquares Means. R package version 1.2.4. CRANR-project.org

Lindström K, Lehtonen TK (2013) Mate sampling and choosiness in the sand goby. Proc Roy Soc B: Biol Sci 280:20130983-20130983

Lindström K, St Mary CM, Pampoulie C (2006) Sexual selection for male parental care in the sand goby, Pomatoschistus minutus. Behav Ecol Sociobiol 60:46-51

Lissåker M, Kvarnemo C (2006) Ventilation or nest defense-parental care trade-offs in a fish with male care. Behav Ecol Sociobiol 60:864-873. https://doi.org/10.1007/s00265-006-0230-0

Malavasi S, Lindström K, Sundström L (2001) Behaviour and success of sneaker males in the sand goby, Pomatoschistus minutus. Acta Ethol 4:3-9. https://doi.org/10.1007/s102110100044

Mazza V, Jacob J, Dammhahn M et al (2019) Individual variation in cognitive style reflects foraging and anti-predator strategies in a small mammal. Sci Rep 9:1-9. https://doi.org/10.1038/s4159 8-019-46582-1

Milinski M, Bakker T (1992) Costs influence sequential mate choice in sticklebacks, Gasterosteus aculeatus. Proc Roy Soc B: Biol Sci 250:229-233

Ogburn MB, Roberts PM, Richie KD et al (2014) Temporal and spatial variation in sperm stores in mature female blue crabs Callinectes 
sapidus and potential effects on brood production in Chesapeake Bay. Mar Ecol Progr Ser 507:249-262

Passos C, Tassino B, Reyes F, Rosenthal GG (2014) Seasonal variation in female mate choice and operational sex ratio in wild populations of an annual fish. Austrolebias reicherti PloS ONE 9:e101649

Pirrone A, Stafford T, Marshall JAR (2014) When natural selection should optimize speed-accuracy trade-offs. Front Neurosci 8:1-5

R Core Team (2018) R: A language and environment for statistical computing.

Reynolds JD, Gross MR (1990) Costs and benefits of female mate choice: Is there a lek paradox? Am Nat 136:230-243. https://doi. org/10.1086/285093

Sullivan MS (1994) Mate choice as an information gathering process under time constraint: implications for behaviour and signal design. Anim Behav 47:141-151

Tang L, Chen J, Ye Z et al (2019) Transcriptomic analysis revealed the regulatory mechanisms of oocyte maturation and hydration in orange-spotted grouper (Epinephelus coioides). Mar Biotechnol 21:537-549. https://doi.org/10.1007/s10126-019-09902-0

Therneau T (2018) coxme: Mixed effects cox models. R package version 2.2-10. https://CRAN.R-project.org/package $=$ coxme, $R$ package version $2.2-10$

Wang M-Y, Chittka L, Ings TC (2018) Bumblebees express consistent, but flexible, speed-accuracy tactics under different levels of predation threat. Front Psychol 9:1601. https://doi.org/10.3389/ fpsyg.2018.01601

Wickelgren WA (1977) Speed-accuracy tradeoff and informationprocessing dynamics. Acta Psychol 41:67-85

Publisher's Note Springer Nature remains neutral with regard to jurisdictional claims in published maps and institutional affiliations. 